\title{
COMPARISON AND TIME SERIES ANALYSIS OF LANDSLIDE DISPLACEMENT MAPPED BY AIRBORNE, TERRESTRIAL AND UNMANNED AERIAL VEHICLE BASED PLATFORMS
}

\author{
J. Pfeiffer ${ }^{1,2 *}$, T. Zieher ${ }^{1}$, M. Rutzinger ${ }^{1,2}$, M. Bremer ${ }^{1,2}$, V. Wichmann ${ }^{3}$ \\ ${ }^{1}$ Institute for Interdisciplinary Mountain Research, Austrian Academy of Sciences, Technikerstr. 21a, 6020 Innsbruck, Austria \\ (thomas.zieher, jan.pfeiffer, martin.rutzinger)@oeaw.ac.at \\ ${ }^{2}$ Institute for Geography, University of Innsbruck, Innrain 52f, 6020 Innsbruck, Austria \\ magnus.bremer@uibk.ac.at \\ ${ }^{3}$ Laserdata GmbH, Technikerstr. 21a, 6020 Innsbruck, Austria \\ wichmann@laserdata.at
}

Commission II, WG II/10

KEY WORDS: Landslide Monitoring, Unmanned Aerial Vehicle Laser Scanning, ULS, Deep-Seated Gravitational Slope Deformation, ICP-Matching, Image Correlation, Displacement Vector Analysis

\begin{abstract}
:
Slow moving deep-seated gravitational slope deformations are threatening infrastructure and economic wellbeing in mountainous areas. Accelerating landslides may end up in a catastrophic slope failure in terms of rapid rock avalanches. Continuous landslide monitoring enables the identification of critical acceleration thresholds, which are required in natural hazard management. Among many existing monitoring methods, laser scanning is a cost effective method providing 3D data for deriving three dimensional and areawide displacement vectors at certain morphological structures travelling on top of the landslide. Comparing displacements between selected observation periods allows the spatial interpretation of landslide acceleration or deceleration. This contribution presents five laser scanning datasets of the active Reissenschuh landslide (Tyrol, Austria) acquired by airborne laser scanning (ALS), terrestrial laser scanning (TLS) and Unmanned aerial vehicle Laser Scanning (ULS) sensors. Three observation periods with acquisition dates between 2008 and 2018 are used to derive area-wide displacement vectors. To ensure a most suitable displacement derivation between ALS, TLS and ULS platforms, an analysis investigating point cloud features within varying search radii is carried out, in order to identify a neighbourhood where common surfaces are represented platform independent or differences between the platforms are minimized. Consequent displacement vector estimation is done by ICP-Matching using morphological structures within the high resolution TLS and ULS point cloud. Displacements from the lower resolution ALS point cloud and TLS point cloud were determined using a modified version of the well-known image correlation (IMCORR) method working with point cloud derived shaded relief images combined with digital terrain models (DTM). The interplatform compatible analyses of the multi-temporal laser scanning data allows to quantify the area-wide displacement patterns of the landslide. Furthermore, changes of these displacement patterns over time are assessed area-wide. Spatially varying areas of landslide acceleration and deceleration in the order of $\pm 15 \mathrm{~cm} \mathrm{a}^{-1}$ between 2008 and 2017 and an area wide acceleration of up to $20 \mathrm{~cm} \mathrm{a}^{-1}$ between 2016 and 2018 are identified. Continuing the existing time series with future ULS acquisitions may enable a more complete and detailed displacement monitoring using entirely represented objects within the point clouds.
\end{abstract}

\section{INTRODUCTION}

Monitoring the movement of deep seated gravitational slope deformations (DSGSDs) is an important tool to detect landslide acceleration and potential slope collapses leading to catastrophic rock avalanches (Carlà et al., 2017, Ostermann and Sanders, 2017). Vegetation penetrating multi-temporal laser scanning has become a cost-efficient data acquisition technique for area-wide change detection analyses (Wujanz et al., 2018) and landslide displacement analyses (Fey et al., 2015, Pfeiffer et al., 2018). Laser scanning exploits the advantageous possibility to remove vegetation with an appropriate filter and allows a more precise displacement analysis focusing on real landslide induced surface changes by neglecting the unintended assessment of vegetation changes. Displacement estimations can be done by using point cloud data representing morphological features travelling on the top of the landslide or by recognizing certain pixel patterns in multi-temporal images derived from point clouds. Object-based

\footnotetext{
*Corresponding author: jan.pfeiffer@oeaw.ac.at
}

point cloud analysis (OBPA) methods are promising approaches where limitations like raster size and raster aggregation method of conventional image based methods can be avoided and a more detailed analysis in landslide monitoring is performable (Mayr et al., 2017). Introducing correspondences between multi-temporal images based on spectral properties and by object-based image analyses (OBIA), may be able to determine displacements, where geometrically based object definitions cannot be introduced.

Displacements are commonly derived between multiple acquisition epochs forming a time series of observation periods. Surface information of each epoch may be assessed with a different acquisition platform demanding the application of interplatform compatible displacement estimation techniques (Corsini et al., 2009, Zieher et al., 2018, Young et al., 2010, Ghuffar et al., 2013). Different laser scanning platforms implicate certain advantages or disadvantages considering data properties or acquisition effort. This contribution presents an existing time series covering 10 years of airborne laser scanning (ALS) and terrestrial laser scanning (TLS) data which is recently continued with Unmanned 

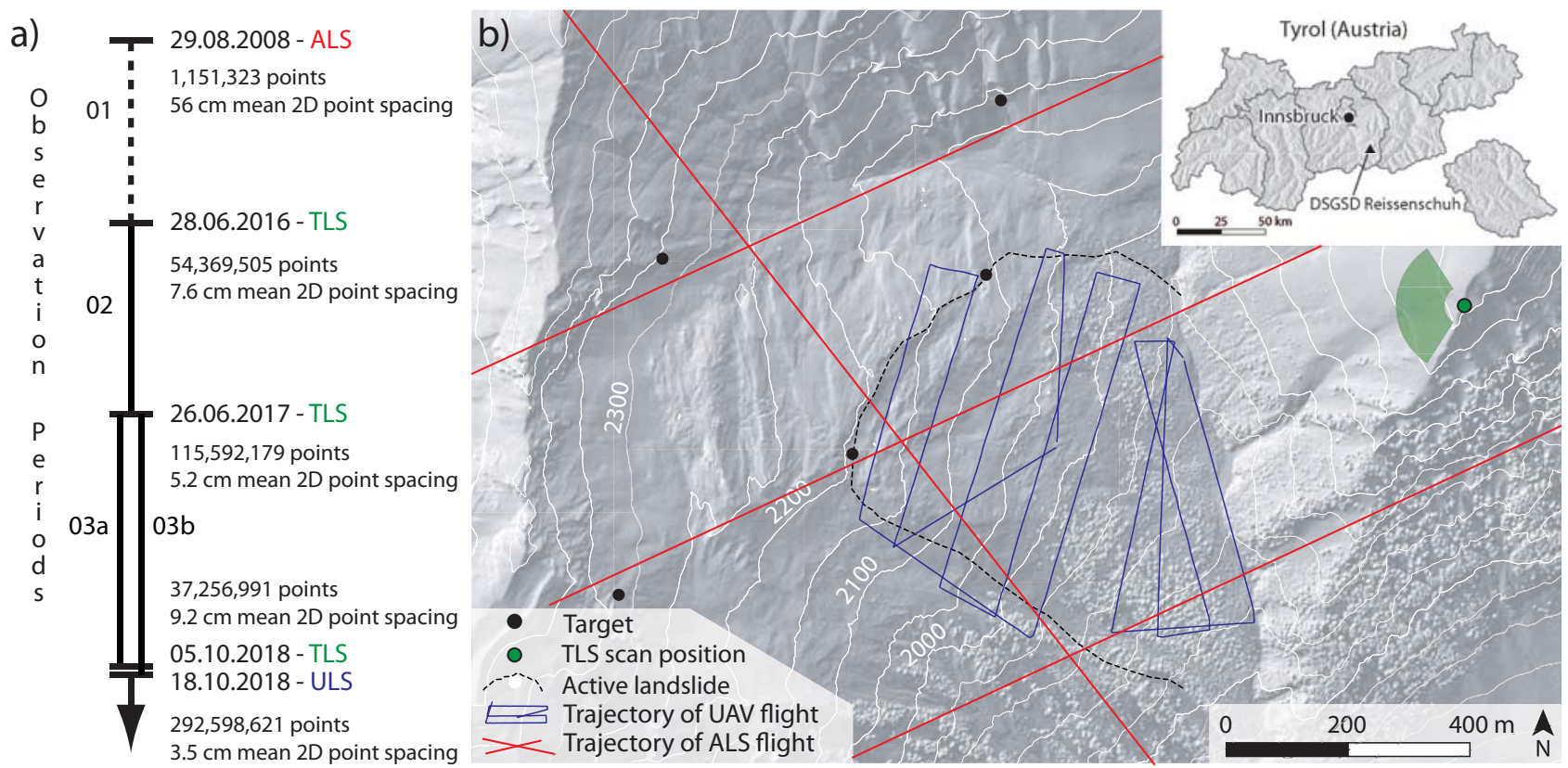

Figure 1. a) Laser scanning datasets with respective characteristics and acquisition dates indicating three observation periods. b) Location of the Reissenschuh landslide in the province of Tyrol, Austria and illustrated description of the laser scanning acquisition setup.

aerial vehicle Laser Scanning (ULS) (Fig. 1a). The objectives of this study are (i) to describe the major differences of point clouds obtained from different laser scanning platforms, (ii) to identify and apply an interplatform compatible method to derive landslide displacements from different laser scanning platforms and (iii) to present and interpret a time series of landslide displacements obtained from an integration of multi-platform laser scanning data indicating landslide movement patterns (e.g. landslide acceleration or deceleration) between different observation periods.

\section{STUDY AREA}

The monitored Reissenschuh landslide can be classified as a DSGSD as mean displacements of $0.62 \mathrm{ma}^{-1}$ were determined in a previous study (Pfeiffer et al., 2018) and geomorphological interpretations of the landslide movement indicate a shear-plane that is located several meters below the surface. The DSGSD is situated on a south facing slope of the east-west striking Schmirn valley (Tyrol, Austria) (Fig. 1b). The currently active part of the DSGSD covers altitudes between 2200 and $1700 \mathrm{~m}$ a.s.l. and is surrounded by summits of up to $2600 \mathrm{~m}$ a.s.l. to the west, north and east. The bedrock surrounding the landslide is made of penninic Bündner schist (Bündnderschiefer) with varying content of lime. The composition of the Bündner schist on the northern slopes of the Schmirn valley is characterized by calcareous rich schists overlaying schists deficient in lime (Frisch, 1979). Embedded within this sequence, some non-calcareous and phyllite-rich layers of black schists containing millimetre-thin quartzite layers are facilitating slope deformations (Rockenschaub et al., 2003).

Lower parts of the landslide are covered by a sparse larch (Larix decidua) and spruce (Picea abies) tree forest while upper parts are dominated by lower vegetation types like Alpine rose (Rhododendron hirsutum), mountain pine (Pinus mugo) or Alpine meadows used for sheep pasture. Beside of vegetation cover, the irregular and humped terrain shows a significant amount of non- vegetated surface features such as bedrock outcrops and randomly distributed single boulders (Fig. 3a) with some larger areas covered by accumulated boulders.

\section{MATERIALS AND METHODS}

\subsection{Airborne Laser Scanning}

ALS data presented in this contribution was commissioned by the Federal state of Tyrol and acquired by TopScan GmbH with an Optech Inc. ALTM3100 system on 29.08.2008. The surface of the Reissenschuh landslide was recorded by four flight strips (Fig. 1b) with approximately $3.1 \mathrm{pts} / \mathrm{m}^{2}$ resulting in an average horizontal point spacing of $56 \mathrm{~cm}$.

\subsection{Terrestrial Laser Scanning}

Three point clouds were acquired using a RIEGL VZ ${ }^{\circledR}-6000$ long-range terrestrial laser scanner form the opposite ridge. Acquisitions were carried out on 28 June 2016, 26 June 2017 and 5 October 2018 using different measurement programs concerning power supply, accessibility and eye safety since the instrument operates a class 3B laser with a wavelength of $1064 \mathrm{~nm}$ and a beam divergence of $0.12 \mathrm{mrad}$. Horizontal respectively vertical angle resolutions vary between $0.003^{\circ}$ and $0.006^{\circ}$ depending on the acquisition date. These settings led to point clouds with approximately 120 to $173 \mathrm{pts} / \mathrm{m}^{2}$ within the active landslide part and an average horizontal point spacing of $7.6 \mathrm{~cm}$ to $9.1 \mathrm{~cm}$. The scanning position was chosen to achieve an optimal coverage of the active landslide part and was estimated beforehand by visibility analysis based on a digital surface model (DSM) derived from the ALS data from 2008. Three 30x30 cm and two $15 \times 15 \mathrm{~cm}$ rectangular reflective targets were installed on stable parts around the landslide to facilitate point cloud registration (Fig. 1b). 


\subsection{Unmanned Aerial Vehicle-based Laser Scanning}

ULS was carried on 18 October 2018 using a RIEGL VUX-1LR laser scanner mounted on a RIEGL RiCOPTER with an Applanix AP20 Inertial Measurement Unit (IMU). Efficient data acquisition, especially in steep and remote terrain, is facilitated by creating a detailed flight plan using the software UgCS (UgCS, 2019). For flight security, 3D distance calculations between projected flight path and DSM surface were carried out. The flight plan was adapted by considering the UAV pitch angle, the angular resolution of the scanner, the pulse repetition rate (PRR) and the flight velocity to optimize point density and coverage of the scanned area. Finally two UAV flights were carried out with flight altitudes ranging between $50 \mathrm{~m}$ and $140 \mathrm{~m}$ above ground and horizontal velocities of $8 \mathrm{~m} / \mathrm{sec}$. An angular resolution of $0.0476^{\circ}$ and a PRR of $820 \mathrm{kHz}$ resulted in a point cloud with $803 \mathrm{pts} / \mathrm{m}^{2}$ and an average horizontal point spacing of $3.5 \mathrm{~cm}$.

\subsection{Data Processing}

Data acquired with the TLS was processed using the RiScan Pro (Riegl LMS, 2019) software to recalculate multiple echoes in the air (multiple time around, MTA) and by using the SAGA GIS (Conrad et al., 2015) software with the LIS Pro 3D (Rieg et al., 2014) extension for further processing steps like registration, georeferencing, tiling, ground filtering, point cloud feature calculation and displacement estimation (Fig. 2). Five rectangular reflector targets with known geometry mounted on stable bedrock around the active landslide were used for registration and georeferencing. The registration was further improved by applying the iterative closest point (ICP) algorithm (Besl and McKay, 1992) on stable, well distributed and non-vegetated areas. To ensure efficient data handling the TLS point clouds were split into $100 \times 100 \mathrm{~m}$ tiles with an overlap of $10 \mathrm{~m}$. Virtual point clouds were created in order to handle and access the point cloud tiles based on a XML file, describing the spatial organisation of the tiles (Wichmann, 2016). A progressive triangular irregular network (TIN) densification approach was applied to every point cloud tile to distinguish between ground and non-ground points (Axelsson, 2000). The classified point clouds were further used to create digital terrain models (DTMs) by only using ground points. Subsequently, shaded relief images were derived from the DTMs using the ambient occlusion algorithm (Tarini et al., 2006).

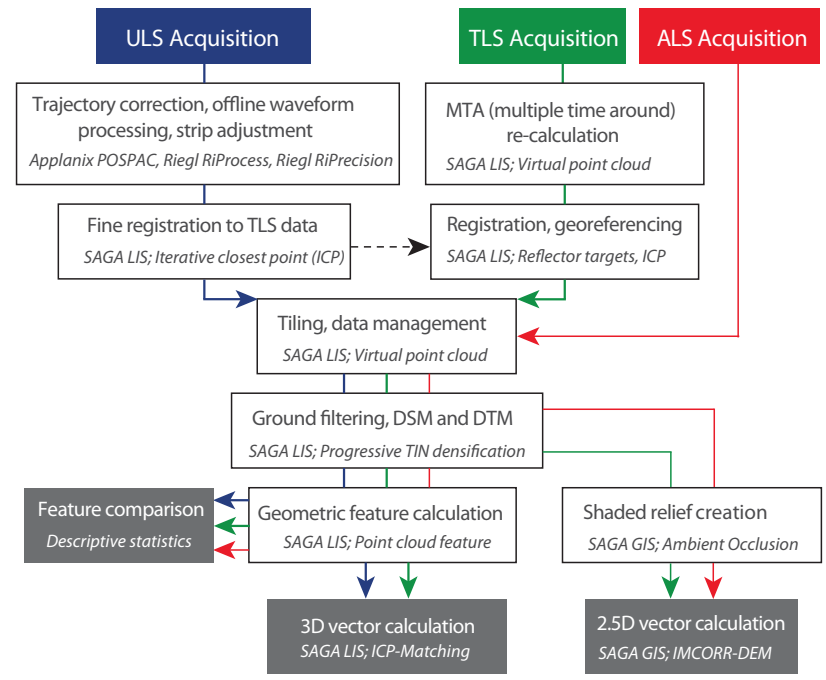

Figure 2. Workflow showing the main data processing steps.
The IMU and Global Navigation Satellite System (GNSS) data of the UAV flight trajectory were differentially corrected using simultaneously recorded reference data from a GNSS base station operated by the South Tyrolean Positioning Service (STPOS, 2019). The software package RiProcess (Riegl LMS, 2019) was used to process the laser scanning data based on the corrected trajectory to create georeferenced point coordinates and the RiPrecision (Riegl LMS, 2019) software package was used for strip adjustment in order to minimize differences between single flight strips. The ULS point cloud was then transformed to the TLS point cloud acquired on 5 October 2018 using stable nonvegetated areas and the ICP algorithm. The same tiled data management as applied to the TLS data was used to enhance further data processing like ground filtering, geometric feature calculation and 3D displacement vector derivation.

The ALS data is provided as an already processed and georeferenced point cloud. After tiling, the same processing steps as applied to the TLS data were carried out.

\subsection{Point Cloud Feature Calculation}

Point cloud features are geometrical features calculated within a spherical neighbourhood for each point. Height, density, local plane and eigenvalue features can be differentiated. Point cloud features are well suited to describe geometrical properties of point clouds in a quantitative way. Consequently, they are useful measures to describe differences in laser scanning data obtained from different platforms. Furthermore, point cloud features describe certain morphologies on the landslide surface where displacement estimation performs well. Locally fitted planes using least-squares fitting within a defined neighbourhood are either described by their normal vector or by aspect and slope angles indicating dip angle and dip direction of the plane. The standard deviation of the distance of all points to the fitted plane is a useful measure to describe the quality of the local fitted plane.

A principle component analysis enables the calculation of eigenvalues and eigenvectors $(e 1, e 2, e 3)$ of the $3 \mathrm{D}$ structure tensor or covariance matrix where the eigenvector $1(e 1)$ is a vector with the largest eigenvalue, the eigenvector $2(e 2)$ is a vector with the medium eigenvalue and eigenvector $3(e 3)$ is a vector with the smallest eigenvalue. Consequently, eigenvectors are useful measures in describing the geometric shape of objects (Gross and Thoennessen, 2006). A constellation of two long eigenvectors ( $e 2$ and $e 1$ ) and one small eigenvector (e3) represents planar surfaces where $e 3$ corresponds to the surface normal vector $(n)$. The omnivariance is a feature that is calculated based on the three eigenvalues within the defined neighbourhood. The omnivariance can be seen as a measure of the roughness. The higher the omnivariance value, the less differences between the eigenvalues and the rougher a surface appears.

$$
\text { omnivarince }=\sqrt[3]{e 1 * e 2 * e 3}
$$

The geometric curvature $M_{\text {curv }}(p)$ of a point $p$ is defined as the average difference between the direction of the local normal vector $n_{p}$ and the direction of the normal vectors $n_{p(j)}$ of $k$ considered neighbouring points $j$ (Bae and Lichti, 2004).

$$
M_{\text {curv }}(p)=\frac{1}{k} \sum_{j=1}^{k}\left\|n_{p}-n_{p(j)}\right\|
$$




\subsection{Landslide Displacement Estimation}

Landslide displacement estimation was done by applying a 3D and a $2.5 \mathrm{D}$ approach. $3 \mathrm{D}$ vectors were derived at morphological features extracted from the TLS and ULS point clouds by using a geometric curvature threshold. Convex and concave morphological structures like block edges, ridges or scarps can be summarized as geomorphological breaklines. Their complex geometrical properties are well suited to introduce multi-temporal correspondence by deriving 3D displacement vectors. Assuming that the features morphology remains the same between the acquisition dates, the ICP-Matching algorithm implemented in the SAGA-LIS software was applied on extracted multi-temporal geomorphological breaklines (Pfeiffer et al., 2018, Wichmann, 2016). The ICP-Matching algorithm is a modified version of the ICP algorithm where $3 \mathrm{D}$ displacement vectors are created by solving the icp problem and connecting the initial slave point positions with their resulting positions after transformation. This is done for each point cloud structure contained within a $10 \times 10 \times 10 \mathrm{~m}$ voxel. The translation is carried out iteratively until a minimum point to surface distance or a defined maximum number of iterations is reached.

The limitations to describe this small-scale geomorphological breaklines using ALS point cloud data (see 4.1) requires the application of another landslide displacement estimation method. The IMCORR-DEM method implemented in the SAGA software (Conrad et al., 2015) can be used to circumvent this issue by utilizing other features for correspondence building (Bremer, 2012). The creation of DTMs from classified point clouds with an adequate cell size allows a further derivation of shaded relief images using the ambient occlusion method (Tarini et al., 2006). The recognition of corresponding pixel patterns in two different shaded relief images derived from DTMs acquired at different epochs enables the horizontal estimation of 2D displacements. Adding the DTM elevation information to the respective corresponding pixels enables the determination of 2.5D displacements. Long term displacement analysis between 2008 and 2018 using one ALS data set, three TLS datasets and one ULS data set were carried out by using either the ICP-Matching method or the IMCORR-DEM method depending on the point cloud properties, the level of detail and their potential to describe complex surfaces. Displacement comparisons were done by assuming a uniform landslide movement throughout a year to calculate annual displacements from the temporal irregularly acquired point clouds.

\section{RESULTS}

\subsection{Surface Representation in ALS, TLS and ULS Point Clouds}

The suitability to describe certain morphological surface structures within point clouds by using geometric features depends on point cloud properties and acquisition platform (ALS, TLS or ULS). As landslide displacements are preferentially quantified by using certain morphological structures within the point clouds, these structures need to be described platform independently. Occlusions are quantified as coverage and the spatial resolution usually specified as point spacing are two main parameters affecting the quality of object representation. A landslide surface subset was used to assess the differences between ULS, TLS and ALS data (Fig. 3 and Table 1).

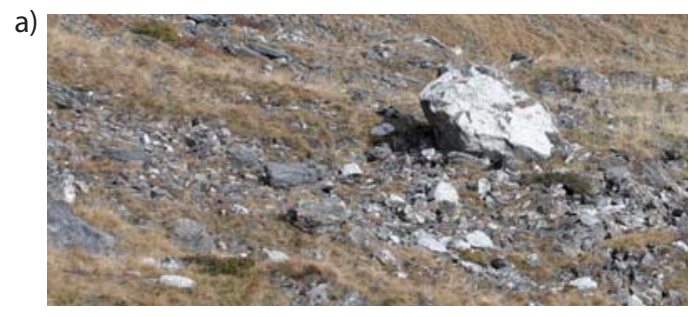

b)
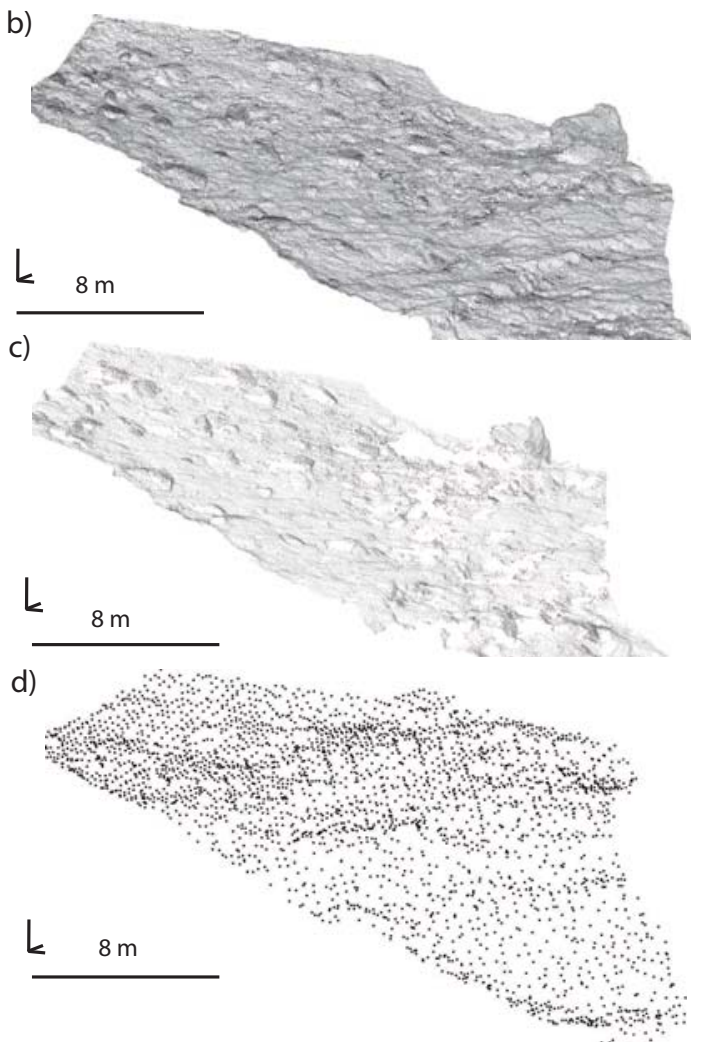

Figure 3. Subset of landslide surface a) photographed during fieldwork and visualised by point clouds acquired with b) ULS, c) TLS and d) ALS

Table 1. Properties of different laser scanning data

\begin{tabular}{lcc}
\hline Platform & Coverage $(\%)$ & Point spacing $(\mathrm{cm})$ \\
\hline ULS & 96.2 & 3.5 \\
TLS & 78.7 & $5.2-9.1$ \\
ALS & 97.7 & 56.2 \\
\hline
\end{tabular}

The ULS point cloud with a mean point spacing of $3.5 \mathrm{~cm}$ describes the surface geometry with a constant point distribution, without major data gaps and with a high level of detail (Fig. 3b). The average point spacing of 5.2-9.1 cm, as it is assumed for the TLS point clouds, is still sufficient to map surface textures, but occlusion effects and irregular point density distribution within the TLS point cloud may lead to an incomplete surface description (Fig. 3c). The point spacing of the ALS point cloud of about $56 \mathrm{~cm}$ makes it impossible to detect smaller morphological structures on the landslide surface (fig. 3d). Thus, a point cloud based determination of landslide displacement using geometrically described features is not feasible. Nevertheless a coverage of $98 \%$ for the ALS data offers a good opportunity to derive landslide displacements using an image based method, where the advantage of using larger and interplatform compatible morphological features in terms of pixel patterns can be exploited. A comparative study 

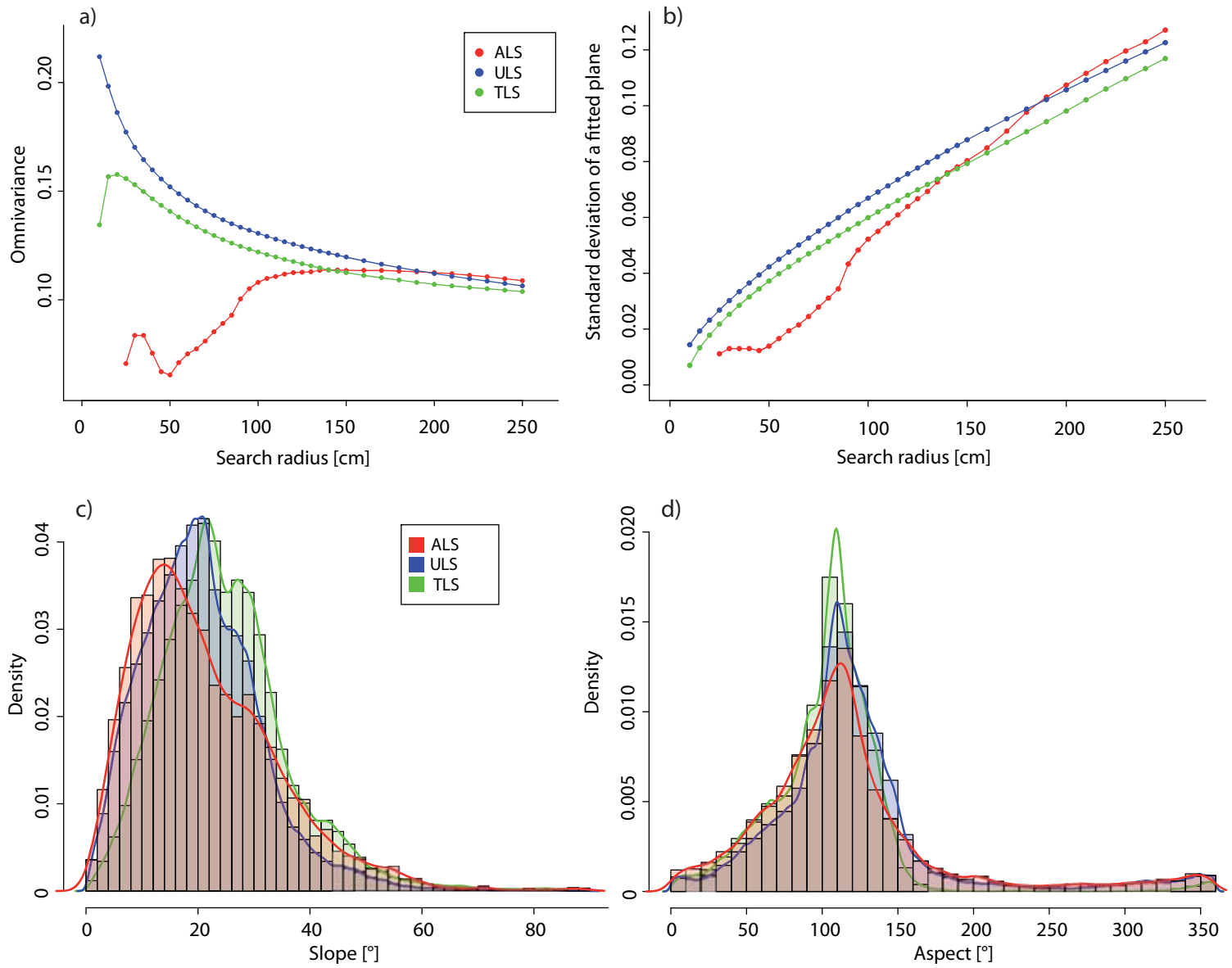

Figure 4. Median (a) omnivariance values and (b) standard deviations of distances from points to the fitted plane calculated by using different search radii for a subset of ALS, ULS and TLS point cloud data. Histograms showing frequency density of (c) slope angles and (d) aspect angles of fitted planes within a $150 \mathrm{~cm}$ spherical neighbourhood occurring within the same point cloud subset but acquired with different laser scanning platforms.

of point cloud features (e.g. omnivariance and standard deviation of a fitted plane) calculated within different search radii enables the identification of a common neighbourhood in which the influence of platform characteristics on displacement derivation can be minimized.

The omnivariance and therefore the surface roughness generally decrease with increasing search radius, except for the ALS data, where an increase from 50 to $150 \mathrm{~cm}$ search radius is followed by an omnivariance decrease towards higher radii. Major differences between ULS and TLS are observable at smaller search radii. With increasing search radius the differences decrease. In general the ULS data features higher omnivariance values, especially at smaller neighbourhoods which suggests them to be rougher and further demonstrates the ability to represent smaller topographic structures compared to the other laser scanning platforms (Fig. 4a.). The maximum omnivariance value per laser scanning platform is reached at different search radii. The radius indicating the highest omnivariance value might correspond to the minimum size of morphological structures that can be described with the respective laser scanning platform.

The median standard deviations of distances from points to the fitted plane for the whole point cloud subset behave similar for each laser scanning platform. With increasing search radius, which is used for the plane fitting, the standard deviations in- crease. The ULS data generally features higher standard deviations compared to the TLS and ALS point cloud at smaller search radii (Fig. 4b.). At larger search radii the standard deviation of the ALS point cloud exceeds the ULS and TLS values, which might be associated with much lower point distances.

The search radius at which the smallest differences of omnivariance and standard deviation of fitted planes between the platforms occur, indicates a neighbourhood where the planes described by different platforms are almost similar. This assumption can be confirmed by comparing surface parameters such as aspect and inclination of locally fitted planes using the same spherical neighbourhood of $150 \mathrm{~cm}$ (Fig. 4c and d).

\subsection{Long Term Displacement Analysis}

Applying a displacement derivation method adapted to the properties of the existing data allows the exploitation of ten years of laser scanning observation history. Three observation periods given by five laser scanning acquisitions are examined and compared. Based on the results gained from a point cloud property analysis (see 4.1) displacement vectors were derived from the ALS data from 29 August 2008 and the TLS data from 28 June 2016 (period01) by using the IMCORR-DEM approach. Displacement vectors were also derived from the TLS data from 28 June 2016 and 26 June 2017 (period02) and the TLS data from 26 


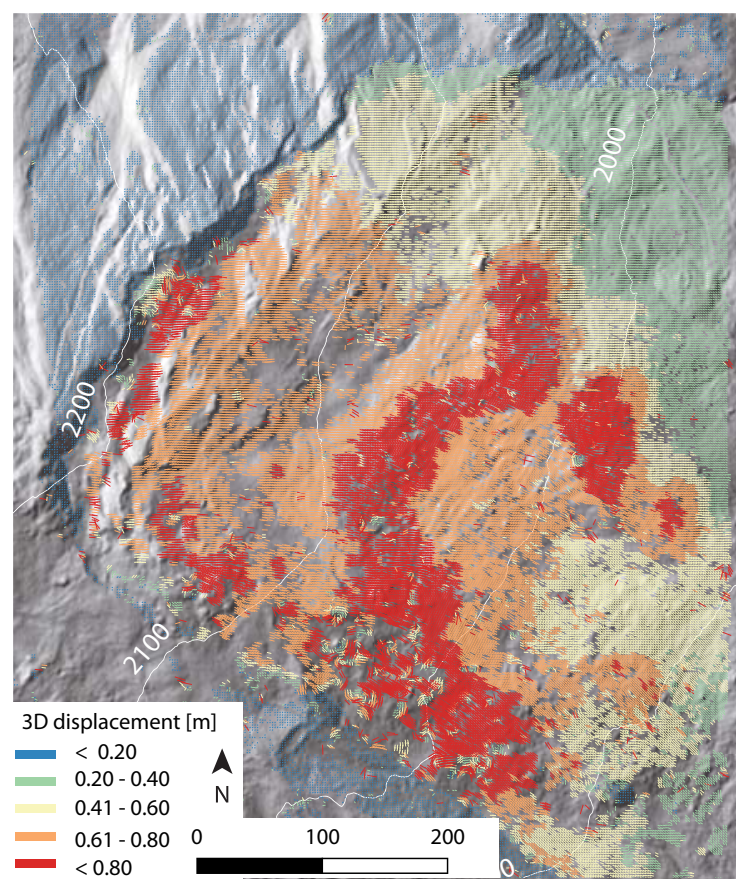

Figure 5. 3D displacement vectors obtained between 28 June 2016 and 26 June 2017 (period02).

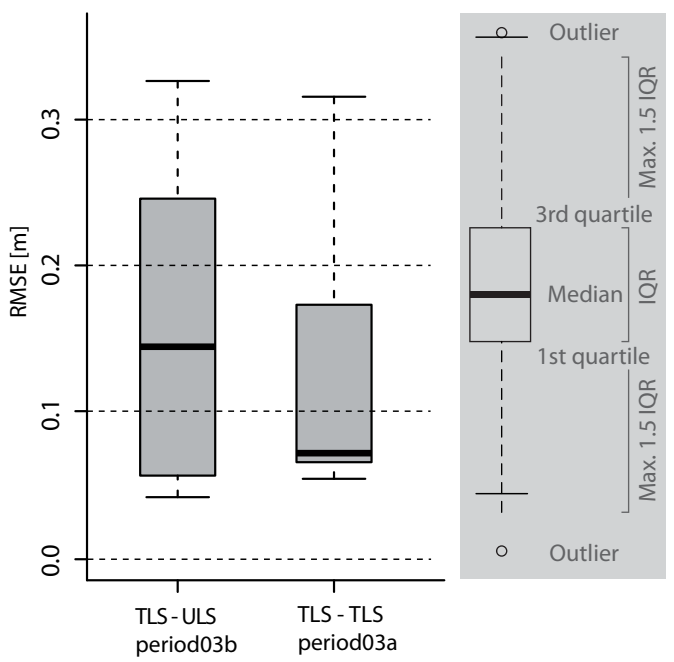

Figure 6. Boxplots comparing root-mean-squared errors

(RMSE) of 3D displacement vectors obtained from ICP-Matching with either TLS to ULS or TLS to TLS data. IQR: Interquartile range.

June 2017 and 5 October 2018 (period03a) as well as the UAVLS data from 18 October 2018 (period03b) by exploiting the ICPMatching method. Geomorphological breaklines extracted by using a geometric curvature threshold calculated within a $50 \mathrm{~cm}$ spherical neighbourhood were used as objects to quantify displacements. The derived vectors were spatially aggregated to median displacement and direction values within a $2 \times 2 \mathrm{~m}$ grid to illustrate the spatial pattern of the displacement vectors distributed over the landslide. A similar pattern of displacement magnitude and direction between all observation periods can be observed. Figure 5 illustrates this pattern occurring within observation pe- riod02 representative for all other observation periods. How well the corresponding morphologies are rotated and transformed to each other after applying the ICP-Matching approach is recorded by the root-mean-squared error (RMSE) attached as an attribute to every point in the transformed point cloud. Observation period 03a is obtained from two TLS acquisitions and observation period $03 \mathrm{~b}$ from one TLS acquisition and one ULS acquisition. As they cover almost the same time span, differences of the vector quality using the RMSE values are compiled (Fig. 6). Lower and less scattered RMSE values between the two TLS acquisition are apparent, where higher and wider scattered RMSE values arise within vectors derived from ULS and TLS data indicating more mistakenly matched morphological structures.

A simple spatial comparison of displacement vectors covering different observation periods is done by a subtraction of annual 3D displacements. Maps of differential displacements indicate areas of landslide acceleration or deceleration. A general landslide acceleration of up to $20 \mathrm{~cm} \mathrm{a}^{-1}$ is observable by comparing the annual displacement vectors obtained between period02 and period03 (Fig. 7a). A pattern of deceleration and acceleration in the order of $\pm 15 \mathrm{~cm} \mathrm{a}^{-1}$ is identified between period01 and period02, where areas of deceleration prevail (Fig. 7b). A comparison of period01 with period03 shows similar pattern of acceleration and deceleration but with more areas showing acceleration instead of deceleration (Fig. 7c).

\section{DISCUSSION}

The comparison of point cloud properties acquired with different laser scanning platforms shows discussable advantages and disadvantages regarding their ability to derive landslide displacement vectors. ALS platforms provide point clouds with a good coverage that may cover much larger areas than those which can be acquired with TLS or ULS platforms. Compared to 2D image data without elevation (e.g. satellite or aerial images), images derived from ALS data have the ability to exploit the IMCORRDEM approach with additional height information to derive 2.5D displacement vectors instead of pure 2D vectors. Nevertheless, ALS data in this case is not suitable to describe complex surfaces in a high level of detail. Morphological structures smaller than $150 \mathrm{~cm}$ cannot be described by using the ALS point cloud. Furthermore, the data is prone to describe horizontal areas in more detail than vertical areas. Point cloud acquisition with TLS platforms from multiple scanning positions with small scanning distances to minimize shadowing effects and to provide homogeneous point clouds with high measurement accuracy is often limited due to complex terrain and the accessibility of areas affected by geomorphological processes like landslides. Considering these issues, only one scan position was used within this case study, implicating advantages and disadvantages compared to the ALS data. Smaller point spacing and higher point densities at vertical structures allow a more precise detection of morphological features within the TLS point cloud, where issues like incomplete coverage and smaller acquisition areas are apparent. ULS links the ALS advantages of good coverage and homogeneous point densities with the advantage of the TLS data like smaller point spacing facilitating a more precise description of morphologies with a high level of detail.

Quality differences between vectors derived from ULS and TLS respectively only TLS may occur due to different surface representations of ULS and TLS data. As 3D vectors were derived by using the ICP-Matching on morphological structures extracted 

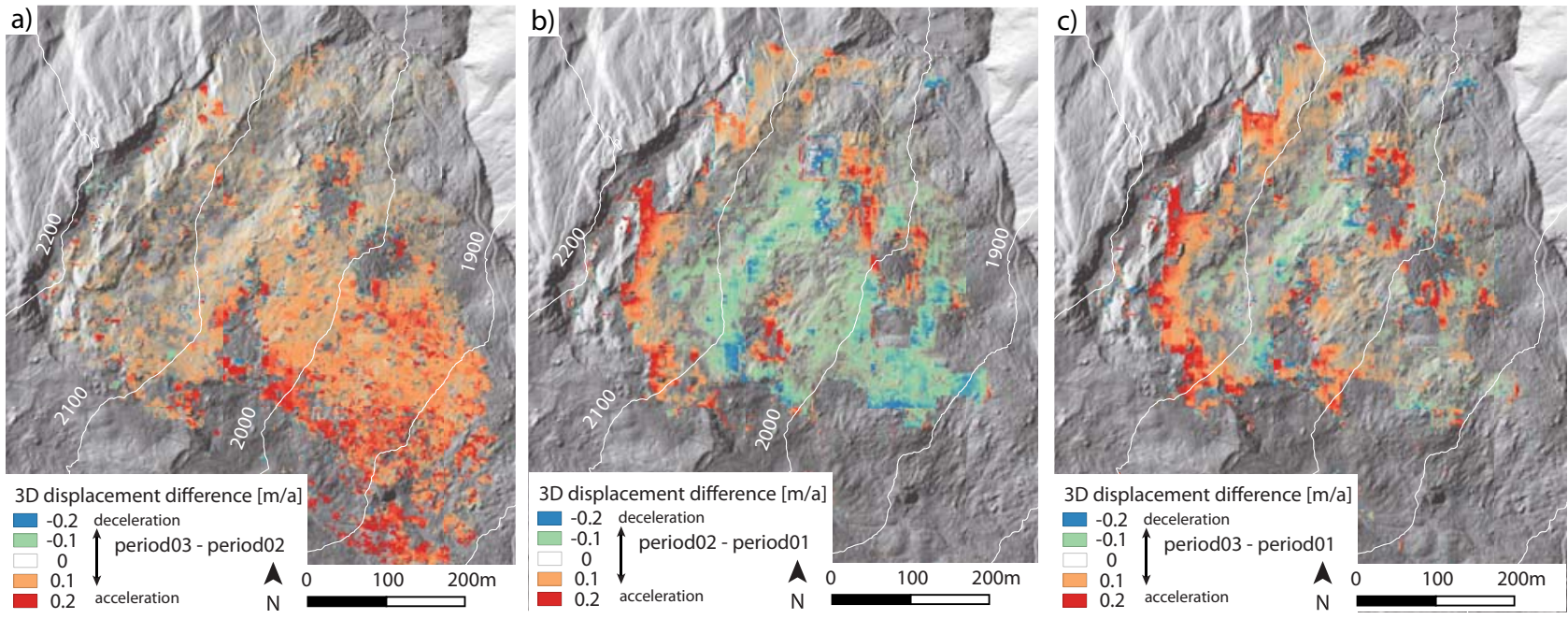

Figure 7. 3D displacement differences indicating areas of acceleration or deceleration based on annual displacements derived between

(a) period03 (26 June 2017-05 October 2018) and period02 (28 June 2016-26 June 2017), between (b) period02 (28 June $2016-26$

June 2017) and period01 (29 August 2008-28 June 2016) and between (c) period03 (26 June 2017-05 October 2018) and period01 (29 August 2008-28 June 2016).

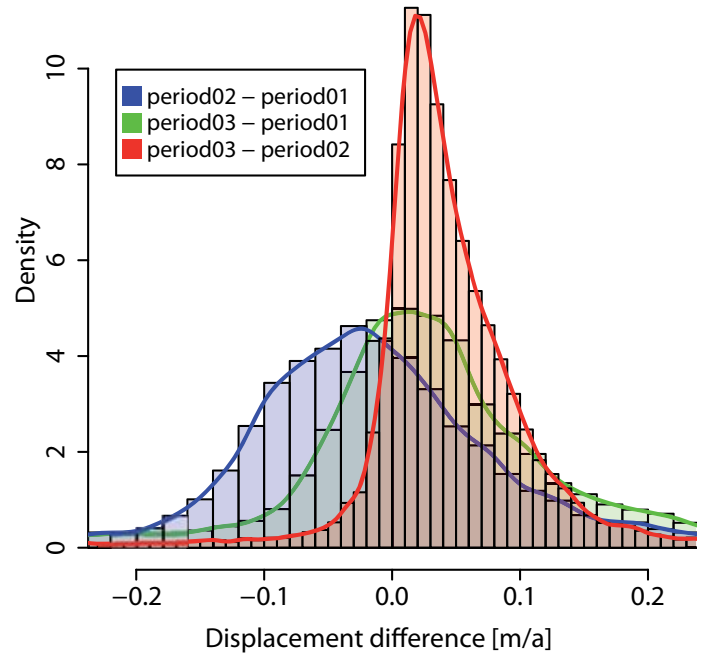

Figure 8. Frequency density distributions of annual displacement differences obtained by subtracting displacement vectors acquired within different observation periods.

by a certain geometric curvature threshold, the representation of these structures may differ regarding the laser scanning platform used for acquisition. The transformation of the extracted ULS surface morphologies to the extracted TLS surface morphologies causes more errors than the transformation of point clouds acquired with the same platform. Adapting the geometric curvature threshold of the ULS point cloud may enable a better matching of their morphologies with them obtained from TLS.

Two potentially result influencing issues were neglected in identifying areas of acceleration and deceleration within the investigated time series. First, identified accelerations and decelerations in the investigated time series are affected by the assumption of continuous landslide movement throughout a year. Annual displacements were derived from irregularly distributed acquisitions throughout the time. Second, the compared landslide displacement vectors were derived using either a $2.5 \mathrm{D}$ or a $3 \mathrm{D}$ approach which can be a source of differences by itself. Nevertheless, this method offers a unique possibility to identify area-wide spatial differences of the landslide behaviour over the time.

\section{CONCLUSION AND OUTLOOK}

Major differences between point clouds acquired from ALS, TLS and ULS regarding spatial resolution and coverage are evident which further affect point cloud derived geometrical attributes like the orientation and standard deviation of fitted planes and the omnivariance of a point within a spherical neighbourhood. The orientation of these planes varies between the platforms. The differences can be minimized within a neighbourhood of about $150 \mathrm{~cm}$. The analysis of roughness parameters within a varying search radius enabled the determination of a minimum size of morphological structures for each laser scanning platform indicating if a displacement estimation based on correspondences of geometrical features is feasible. TLS and ULS data turned out to be well suited for geometrical based displacement derivations, where the ALS data is not able to describe the same morphological structures as they occur within the TLS and ULS data. As a consequence, displacement vectors derived by the ALS data were created by using the IMCORR-DEM approach.

Handling the data differences of the different laser scanning platforms by applying an adequate method for displacement estimation finally enables the determination of landslide movement patterns throughout the past ten years. Annual landslide displacement differences in the order of $\pm 15 \mathrm{~cm} / \mathrm{a}$ between 2008 and 2017 indicate areas of landslide acceleration and deceleration whereas annual displacement differences between 2016 and 2018 demonstrate an area wide acceleration of up to $20 \mathrm{~cm} / \mathrm{a}$. Differences in describing complex morphologies between ULS and TLS data cause displacement vectors with higher errors related to poor surface matching. Developing a relation translating ULS described morphologies to TLS described morphologies might overcome this issue in future. The assumption of uniform landslide movement throughout the year has to be verified in future by continuously measuring the landslide displacement with an adequate sensor (e.g. permanently measuring DGNSS logger or 
a permanently installed laser scanner). A second ULS acquisition in 2019 may further enable a more complete and accurate monitoring of the 3D-landslide movement using even smaller and more accurately represented morphologies.

\section{ACKNOWLEDGEMENTS}

The present study was conducted within the OPERANDUM project. This project has received funding from the European Unions Horizon 2020 research and innovation programme under grant agreement No 776848. The Unmanned aerial vehicle Laser Scanning data has been acquired by the 4D LiDAR mountAin Monitoring laB (https://4dlamb.mountainresearch.at/) funded by the Federal Ministry of Education, Science and Research, Republic of Austria.

\section{REFERENCES}

Axelsson, P., 2000. DEM generation from laser scanner data using adaptive TIN models. International Archives of Photogrammetry and Remote Sensing 33(4), pp. 110-117.

Bae, K. and Lichti, D., 2004. Automated Registration of Unorganised Point Clouds from Terrestrial Laser Scanners. In: P. O. Altan (ed.), XXth ISPRS Congress: Geo-Imagery Bridging Continents, pp. 1-6.

Besl, P. J. and McKay, N. D., 1992. A method for registration of 3-D shapes. IEEE Transactions on pattern analysis and machine intelligence 14(2), pp. 239-256.

Bremer, M., 2012. IMCORR - Feature Tracking. SAGA Module Reference. http://www.saga-gis.org.

Carlà, T., Intrieri, E., Di Traglia, F., Nolesini, T., Gigli, G. and Casagli, N., 2017. Guidelines on the use of inverse velocity method as a tool for setting alarm thresholds and forecasting landslides and structure collapses. Landslides 14(2), pp. 517-534.

Conrad, O., Bechtel, B., Bock, M., Dietrich, H., Fischer, E., Gerlitz, L., Wehberg, J., Wichmann, V. and Böhner, J., 2015. System for automated geoscientific analyses v. 2.1.4. Geoscientific Model Development 8(7), pp. 1991-2007.

Corsini, A., Borgatti, L., Cervi, F., Dahne, A., Ronchetti, F. and Sterzai, P., 2009. Estimating mass-wasting processes in active earth slides earth flows with time-series of high-resolution dems from photogrammetry and airborne lidar. Natural Hazards and Earth System Sciences 9(2), pp. 433-439.

Fey, C., Rutzinger, M., Wichmann, V., Prager, C., Bremer, M. and Zangerl, C., 2015. Deriving 3D displacement vectors from multi-temporal airborne laser scanning data for landslide activity analyses. GIScience \& Remote Sensing 52(4), pp. 437-461.

Frisch, W., 1979. Bericht über geologische aufnahmen im tauernfenster auf blatt 148. Verhandlungen der Geologischen Bundesanstalt.

Ghuffar, S., Székely, B., Roncat, A. and Pfeifer, N., 2013. Landslide displacement monitoring using $3 \mathrm{~d}$ range flow on airborne and terrestrial lidar data. Remote Sensing 5(6), pp. 2720-2745.

Gross, H. and Thoennessen, U., 2006. Extraction of lines from laser point clouds. In: Symposium of ISPRS Commission III: Photogrammetric Computer Vision PCV06. International Archives of Photogrammetry, Remote Sensing and Spatial Information Sciences, Vol. 36, pp. 86-91.
Mayr, A., Rutzinger, M., Bremer, M., Oude Elberink, S., Stumpf, F. and Geitner, C., 2017. Object-based classification of terrestrial laser scanning point clouds for landslide monitoring. The Photogrammetric Record 32(160), pp. 377-397.

Ostermann, M. and Sanders, D., 2017. The benner pass rock avalanche cluster suggests a close relation between long-term slope deformation (dsgsds and translational rock slides) and catastrophic failure. Geomorphology 289, pp. 44 - 59.

Pfeiffer, J., Zieher, T., Bremer, M., Wichmann, V. and Rutzinger, M., 2018. Derivation of Three-Dimensional Displacement Vectors from Multi-Temporal Long-Range Terrestrial Laser Scanning at the Reissenschuh Landslide (Tyrol, Austria). Remote Sensing 10(11), pp. 1688.

Rieg, L., Wichmann, V., Rutzinger, M., Sailer, R., Geist, T. and Sttter, J., 2014. Data infrastructure for multitemporal airborne lidar point cloud analysis examples from physical geography in high mountain environments. Computers, Environment and Urban Systems 45, pp. 137 - 146.

Riegl LMS, 2019. http://www.riegl.com/. Last accessed: 17 January 2019

Rockenschaub, M., Kolenprat, B. and A., N., 2003. Das westliche Tauernfenster. Geologische Bundesanstalt - Arbeitstagung 2003: Blatt 148 Brenner.

STPOS, 2019. http://www.stpos.it/SpiderWeb/frmIndex.aspx. Last accessed: 17 January 2019.

Tarini, M., Cignoni, P. and Montani, C., 2006. Ambient occlusion and edge cueing for enhancing real time molecular visualization. IEEE transactions on visualization and computer graphics.

UgCS, 2019. https://www.ugcs.com/. Last accessed: 17 January 2019.

Wichmann, V., 2016. Laserdata LIS Command Reference.

Wujanz, D., Avian, M., Krueger, D. and Neitzel, F., 2018. Identification of stable areas in unreferenced laser scans for automated geomorphometric monitoring. Earth Surface Dynamics 6(2), pp. 303-317.

Young, A. P., Olsen, M., Driscoll, N., Flick, R., Gutierrez, R., Guza, R., Johnstone, E. and Kuester, F., 2010. Comparison of airborne and terrestrial lidar estimates of seacliff erosion in southern california. Photogrammetric Engineering \& Remote Sensing 76(4), pp. 421-427.

Zieher, T., Toschi, I., Remondino, F., Rutzinger, M., Kofler, C., Mejia-Aguilar, A. and Schlögel, R., 2018. Sensor-and sceneguided integration of tls and photogrammetric point clouds for landslide monitoring. International archives of the photogrammetry, remote sensing \& spatial information sciences. 